\title{
ESTUDO DA EFICIÊNCIA DO COMPOSTO ATIVO DE MORINGA OLEIFERA EXTRAÍDA COM SOLUÇÕES SALINAS NA TRATABILIDADE DE ÁGUAS RESIDUÁRIAS DA INDÚSTRIA DE LATICÍNIOS
}

\author{
Dalila Maria Formentini-Schmitt ${ }^{1}$ \\ Márcia Regina Fagundes-Klen ${ }^{1}$ \\ Márcia Teresinha Veit ${ }^{1}$ \\ Rosângela Bergamasco ${ }^{2}$ \\ Anandyara Thais Ferrandin ${ }^{1}$
}

\begin{abstract}
Resumo: A indústria de laticínios é caracterizada pela geração de despejos com altas concentrações de nutrientes, sólidos em suspensão e gorduras, que são tratados por técnicas tradicionais que utilizam coagulantes inorgânicos. Alternativamente estão sendo estudados coagulantes naturais como o extraído das sementes de Moringa oleífera (M. oleífera), que é biodegradável, de baixo custo e gera volumes menores de lodo. A extração do coagulante com solução salina concentrada $(1,0 \mathrm{M})$ eleva a capacidade de coagulação. A carência de alternativas de baixo custo e ambientalmente corretas para tratamento de águas residuárias provenientes das indústrias de pequeno e médio porte motivou este estudo, cujo objetivo é avaliar a eficiência do processo de coagulação/floculação em termos de remoção de cor aparente, turbidez e demanda química de oxigênio (DQO) utilizando a semente de $M$. oleífera e soluções salinas distintas $(\mathrm{KCl} 1,0 \mathrm{M}$ e $\mathrm{NaCl} 1,0 \mathrm{M})$ para extração do coagulante; além de avaliar o comportamento do pH durante o tempo de sedimentação. Os ensaios foram conduzidos em "Jar Test", e foram estudadas as faixas de concentração de 1300 a 1800 ppm de $M$. oleífera em solução salina. Os melhores resultados encontraram-se quando se utilizaram1500 ppm da solução coagulante extraída com $\mathrm{KCl}$ 1,0 M; obteve-se uma remoção de 98,3\% de cor aparente, $97,1 \%$ de turbidez e 58,9\% de DQO.
\end{abstract}

Palavras-chave: coagulação/floculação,efluente de laticínios, coagulante natural.

\begin{abstract}
The dairy industry is characterized by the generation of effluents with high concentrations of nutrients, suspended solids and fats, which are treated by traditional techniques using inorganic coagulants. Alternatively coagulants are being studied as the extracted natural seed Moringaoleifera (M. oleifera), which is biodegradable, low cost and generates minor amounts of sludge. The extraction of the concentrated coagulant saline $(1.0 \mathrm{M})$ increased the coagulation. The lack of alternative low cost and environmentally sound treatment of wastewater from the industries of small and medium-sized motivated this study, whose objective is to evaluate the efficiency of coagulation / flocculation in terms of removal of apparent color, turbidity and demand chemical oxygen demand (COD) using the seed of $M$. oleifera is different salt solutions $(1.0 \mathrm{M} \mathrm{KCl}$ and $1.0 \mathrm{M} \mathrm{NaCl})$ for extraction of the coagulant, in addition to evaluate the behavior of the $\mathrm{pH}$ during the settling time. The tests were conducted in "Jar Test", the concentration ranges from 1300 to $1800 \mathrm{ppm}$ of $M$. oleifera were studied in saline solution. The best results were found when using $1500 \mathrm{ppm}$ of the coagulant solution extracted with $1.0 \mathrm{M} \mathrm{KCl}$; gave a $98.3 \%$ removal of color apparent turbidity of $97.1 \%$ and $58.9 \%$ of the COD.
\end{abstract}

Keywords: coagulation/flocculation, dairy effluent, natural coagulant.

${ }^{1}$ Programa de Pós Graduação Eng. Química - Universidade Estadual do Oeste do Paraná. e-mail: dalila_formentini@hotmail.com

${ }^{2}$ Programa de Pós Graduação Eng. Química - Universidade Estadual de Maringá.

ENGEVISTA, V. 16, n. 2, p.221-231, Junho 2014 


\section{INTRODUÇÃO}

A indústria de alimentos além do consumo de grandes volumes de água potável (Galambos et al., 2004) ainda apresenta problemas relacionados à produção de águas residuárias com altas cargas orgânicas e geração de grandes volumes de lodo (Ramjeawon, 2000).

A indústria de laticínios se insere neste contexto, pois seus despejos são caracterizados por apresentarem elevadas concentrações de nutrientes, sólidos em suspensão e gorduras (Kushwaha et al., 2010). O tratamento inadequado destes despejos pode causar graves danos ambientais.

Estima-se que ocorra um desperdício de $2 \%$ do volume total de leite processado, que por falhas operacionais entre outras causas acabam se misturando ao efluente produzido nos processos de partidas, paradas e lavagem de equipamentos e pisos. O volume dos despejos desta indústria pode variar de 0,2 a10 litros de águas residuárias por litro de leite processado (Tchamango et al., 2010).

Para Kushwaha et al. (2010), as técnicas de tratamento para as águas residuárias da indústria de laticínios geralmente estão associadas aos processos tradicionais que combinam tratamento físico (ou fisico-químico) ao tratamento biológico.

Os coagulantes inorgânicos mais conhecidos e utilizados no tratamento fisico-químico de água e água residuária são os sais de ferro e alumínio, ambos são dependentes de fatores como concentração, $\mathrm{pH}$ e temperatura para que seu desempenho seja satisfatório (Renault et al., 2009).

Muitos estudos já relataram os problemas associados ao uso dos coagulantes inorgânicos à base de sais de ferro e alumínio (Ndabigengesere et al., 1995;Ndabigengesere e Narasiah, 1998), isso tem motivado pesquisadores em buscar alternativas ao uso destes coagulantes.
$\mathrm{O}$ uso de coagulantes naturais no tratamento de água superficial para produção de água potável já ocorre em países como Japão, China, Índia e Estados Unidos (Madrona et al., 2010).

Um dos coagulantes naturais mais estudados atualmente é extraído das sementes de $M$. oleífera, planta tropical, originária da Índia. Atualmente a $M$. oleífera é encontrada em diversos países de clima tropical (Bhatia et al., 2007), inclusive no Brasil.

O uso do coagulante natural extraído das sementes de $M$. oleífera apresenta vantagens importantes em relação ao uso dos coagulantes inorgânicos. Katayon et al. (2007) relataram que os volumes de lodo gerados são menores comparados aos produzidos por coagulantes inorgânicos como os sais de alumínio, além de ser biodegradável e apresentarbaixo risco ao meio ambiente. Bhuptawat et al. (2007) ressaltaram que esse lodo, após estabilização, pode ser utilizado como fertilizante ou condicionador de solos.

Silva et al. (2012),em seus estudos de lixiviação e solubilização dos lodos de policloreto de alumínio (PAC) e Moringa oleifera gerados no processo de coagulação/floculação de água bruta, classificaram estes resíduos como CLASSE II A, Não perigoso - Não inerte. Os autores destacam a necessidade de adequada disposição deste lodo de modo a não causar danos ao meio ambiente e aos seres humanos.

Ndabigengesere et al. (1995) afirmaram que a atividade das sementes de $M$. oleífera como coagulante se deve à presença de proteínas catiônicas solúveis em água. Segundo Okuda et al. (1999), a extração do coagulante, a partir das sementes de $M$. oleífera, utilizando soluções salinas concentradas, pode elevar a capacidade de coagulação em até 7,4 vezes em relação ao coagulante extraído apenas com água destilada.

Schmitt et al. (2012) utilizaram a Moringa oleífera extraída com solução de $\mathrm{KCl} 1,0 \mathrm{M}$ para tratamento de águas 
residuárias da indústria de laticínios e obtiveram remoções de cor aparente e turbidez de $84,5 \%$ e $98,0 \%$, respectivamente. Este resultado demonstra o potencial de uso deste coagulante natural no tratamento deste tipo de efluente.

Alguns estudos relataram a utilização do extrato de $M$. oleífera como coagulante para tratamento de águas com elevada turbidez (Ndabigengesere et al., 1995;Nkurunziza et al., 2009; Madrona et al., 2010) e também para abrandamento de águas duras (Muyibie Evison, 1995).Outros estudos utilizaram este coagulante para tratamento de águas residuárias (Bhatia et al., 2007; Prasad, 2009). Entretanto, existe uma carência de estudos com coagulantes naturais para tratamento de águas residuárias provenientes de agroindústrias como a de laticínios que estão presentes em praticamente todas as regiões do Brasil; normalmente são indústrias de pequeno e médio porte que necessitam de técnicas de baixo custo para tratamento de seus despejos.

Dentro deste contexto, o presente estudo destaca como objetivos: avaliar as eficiências das soluções coagulantes de $M$. oleífera extraídas com soluções salinas distintas de $\mathrm{KCl} 1,0 \mathrm{M}$ e $\mathrm{NaCl} 1,0$ $\mathrm{M}$ em termos de redução de cor aparente, turbidez e DQO, nos processos de coagulação/floculação/sedimentação para a água residuária da indústria de laticínios; avaliar o comportamento do pH durante a sedimentação; além de determinar qual a melhor solução coagulante e sua concentração ideal.

\section{MATERIAIS E MÉTODOS}

\subsection{Caracterização da Água Residuária}

A água residuária utilizada nos ensaios foi coletada na entrada da estação de tratamento de uma indústria de laticínios do Oeste do Estado do Paraná, no Brasil. A mesma foi caracterizada através de ensaios físico-químicos logo após a coleta.Os parâmetros avaliados foram cor aparente e DQO (espectrofotômetro HACH DR/2010), turbidez (turbidímetro HACH $2100 \mathrm{P}$ ), pH (pHmetro Digimed DM-22) e sólidos suspensos totais (APHA, 1994).

Após a caracterização inicial, a água residuária foi homogeneizada, devidamente fracionada e armazenada em condições de congelamento. As amostras necessárias para realização dos ensaios de coagulação/floculação/sedimentação

foram retiradas com antecedência para serem utilizadas à temperatura ambiente $\left(23-25^{\circ} \mathrm{C}\right)$.

\subsection{Preparo da Solução Coagulante de M. Oleífera}

Foram preparadas soluções coagulantes distintas de M.oleífera, uma utilizou solução de $\mathrm{KCl}$ 1,0 M (MO-KCl) e outra utilizou solução de $\mathrm{NaCl}$ 1,0 M (MO-NaCl),segundo Madrona et al.(2010) e Okuda et al.(1999).

As soluções coagulantes foram preparadas para uso imediato. Trituramse em um "blender" $5 \mathrm{~g}$ de sementes de M. oleífera descascadas e $100 \mathrm{~mL}$ de solução de $\mathrm{KCl}$ 1,0 M ou $\mathrm{NaCl}$ 1,0 M. Em seguida esta solução foi mantida sob agitação durante $30 \mathrm{~min}$. e posterior filtração a vácuo(Heredia e SánchezMartín, 2009; Nkurunziza et al., 2009; Madrona et al., 2010).

\subsection{Ensaio de Coagulação/ Floculação/ Sedimentação}

As concentrações utilizadas nos ensaios de coagulação/floculação/sedimentação para as duas soluções coagulantes estudadas (MO-KCl e $\mathrm{MO}-\mathrm{NaCl}$ ) foram de 1300 , $1400,1500,1600,1700$ e 1800 ppm e foram estabelecidas com base na literatura (Bhatia et al., 2007). Não foi necessário ajustar o $\mathrm{pH}$.

Os ensaios de coagulação/floculação/sedimentação

foram realizados em duplicata utilizando um equipamento de "Jar-Test" microcontrolado, marca Milan, modelo JT-103/6. As condições empregadas para $\mathrm{o}$ processo

de 
coagulação/floculação/sedimentação foram velocidade de mistura rápida de $100 \mathrm{rpm}$, tempo de coagulação de $2 \mathrm{~min}$, velocidade de mistura lenta de $20 \mathrm{rpm}$, tempo de floculação de $10 \mathrm{~min}$ e tempo de sedimentação de 60 min (Bhuptawat et al., 2007).

As coletas foram realizadas nos intervalos de 20, 40 e 60 min. Para cada amostra foram determinados os parâmetros $\mathrm{pH}$, cor aparente e turbidez. $\mathrm{O}$ parâmetro DQO foi quantificado apenas nas amostras coletadas no tempo de 60 min. de sedimentação.
A eficiência do processo foi avaliada em termos de remoção de cor aparente, turbidez e DQO.

\section{RESULTADOS E DISCUSSÃO}

\subsection{Caracterização da Água Residuária}

Na Tabela 1 é apresentada uma comparação entre os resultados da caracterização realizada com a água residuária da indústria de laticínios coletada e alguns dados da literatura.

Tabela 1. Caracterização da água residuária e comparação com dados da literatura.

\begin{tabular}{|c|c|c|c|}
\hline Parâmetro & $\begin{array}{c}\text { Resultados } \\
\text { obtidos }\end{array}$ & $\begin{array}{c}\text { Sarkar et al. } \\
(2006)\end{array}$ & $\begin{array}{l}\text { Danalewich } \\
\text { et al. (1998) }\end{array}$ \\
\hline Cor aparente $\left(\mathrm{mgPtCo} \mathrm{L}^{-1}\right)$ & 2541 & - & - \\
\hline Turbidez (NTU) & 711 & $15-30$ & - \\
\hline $\mathrm{pH}(-)$ & 7,59 & $5,5-7,5$ & $1,8-11,3$ \\
\hline DQO $\left(\mathrm{mg} \mathrm{L}^{-1}\right)$ & 2370 & $1500-3000$ & $785-7619$ \\
\hline Sólidos Suspensos Totais $\left(\mathrm{mg} \mathrm{L}^{-1}\right)$ & 540 & $250-600$ & $326-3560$ \\
\hline
\end{tabular}
estudo apresentou expressiva carga orgânica, como pode ser observado pelos valores relativamente elevados dos parâmetros: cor aparente, turbidez, DQO e sólidos suspensos totais.

Apesar da diferença entre os valores de turbidez encontrados comparados aos relatados por Sarkar et al. (2006), conclui-se que o valor encontrado esteja de acordo com as características do local de coleta, pois, segundo Sengile Özakar (2006), cada estabelecimento industrial apresenta características próprias e isso influencia diretamente nos parâmetros físicoquímicos de suas águas residuárias.
Nas Figuras 1 e 2 são apresentados os resultados da variação do $\mathrm{pH}$ durante o tempo de sedimentação e nas diferentes concentrações de coagulante para os ensaios com $\mathrm{MO}-\mathrm{KCl}$ e $\mathrm{MO}-\mathrm{NaCl}$, respectivamente.

Segundo Sarkar et al. (2006), a coagulação/ floculação é uma das etapas mais importantes do tratamento físicoquímico e tem como objetivo a remoção do material em suspensão, responsável pela turbidez das águas residuárias e também pela redução da matéria orgânica que contribui para os índices de demanda biológica de oxigênio (DBO) e DQO presentes nestas águas. 


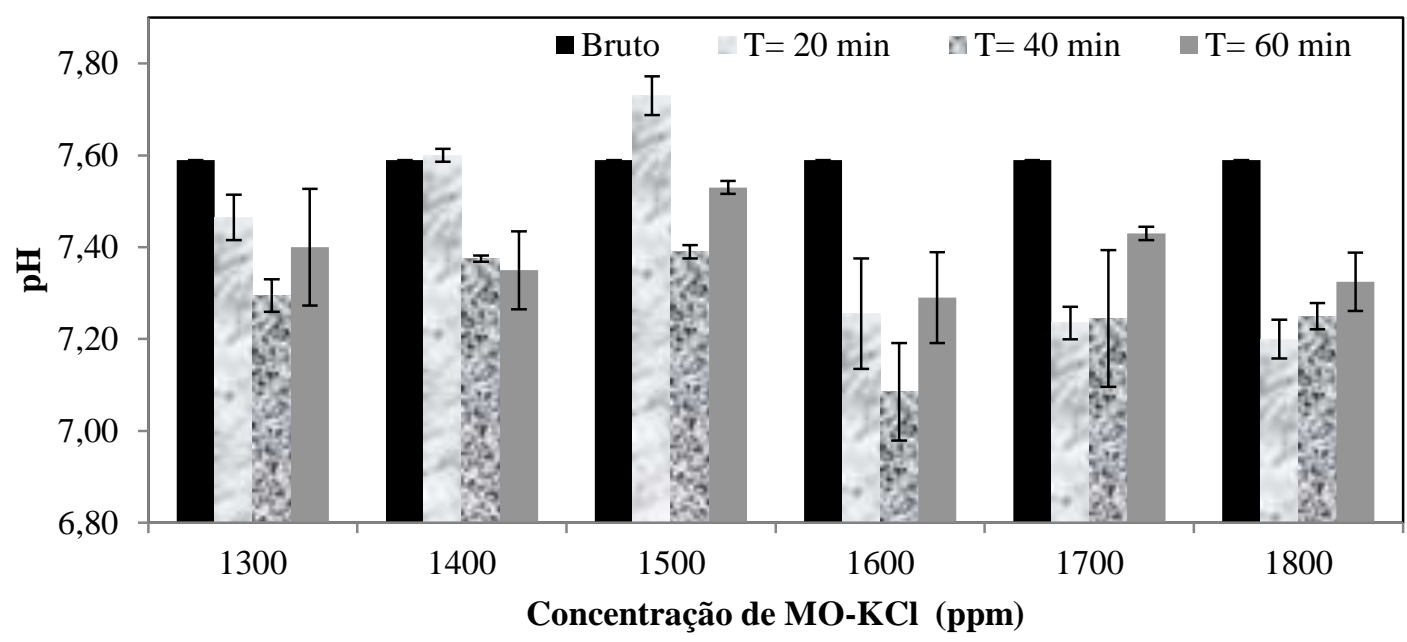

Figura 1. Variação do pH durante o tempo de sedimentação para MO-KCl.

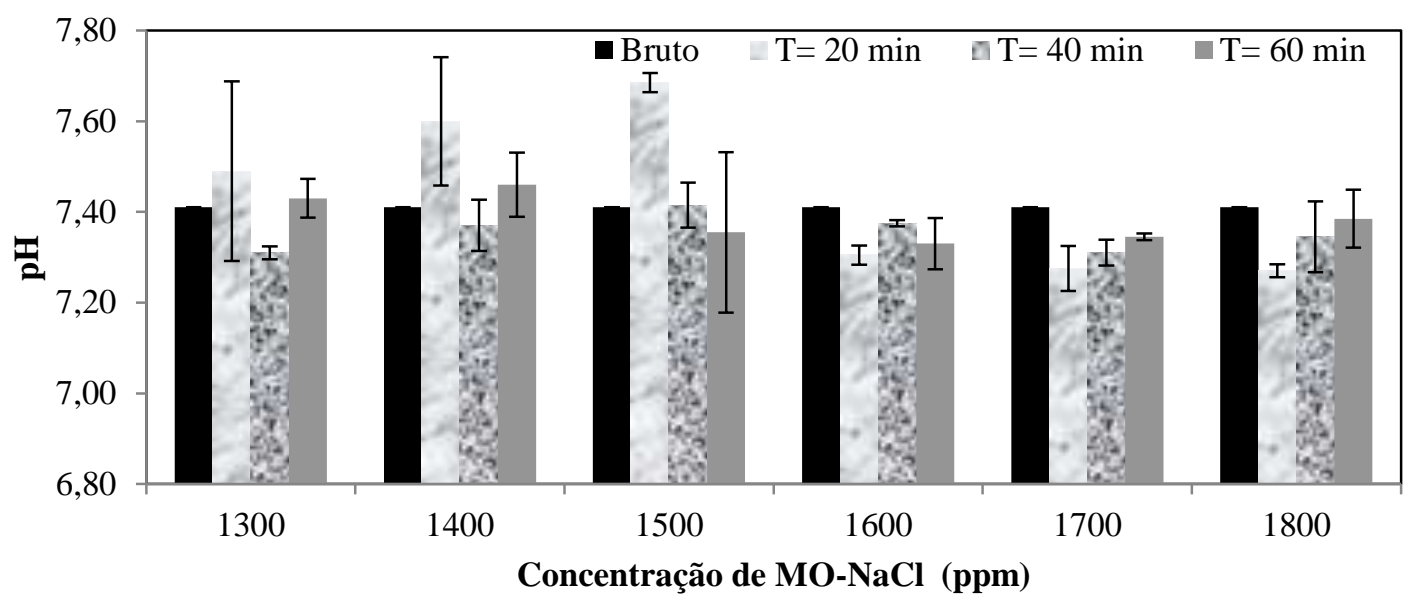

Figura 2. Variação do $\mathrm{pH}$ durante o tempo de sedimentação para $\mathrm{MO}-\mathrm{NaCl}$.

É possível observar nas Figuras 1 e 2 que a variação do $\mathrm{pH}$ entre a amostra de água residuária bruta (antes da adição do coagulante) e após os processos de coagulação/floculação/sedimentação não foi significativa e que o $\mathrm{pH}$ das amostras após o tempo total de sedimentação de 60 min. foi ligeiramente menor que o $\mathrm{pH}$ das mesmas antes do tratamento.A exceção ocorreu nas concentrações de 1300 e 1400 ppm de $\mathrm{MO}-\mathrm{NaCl}$, em que ambas apresentaram variações de $\mathrm{pH}$ nos tempos intermediários e em $\mathrm{T}=60 \mathrm{~min}$. um $\mathrm{pH}$ ligeiramente maior que o $\mathrm{pH}$ da amostra antes do tratamento (bruta). Este fato pode ter relação com a formação de flocos muito pequenos pela baixa concentração do coagulante MO-Na
Clutilizada, o que também ocasionou baixa remoção de cor aparente e turbidez nestas duas concentrações de $\mathrm{MO}-\mathrm{NaCl}$.

Prasad (2009) obteve resultados semelhantes ao estudar a remoção de cor da água residuária de destilaria de álcool, no qual utilizou sementes de $M$. oleífera como coagulante. O ligeiro decréscimo do $\mathrm{pH}$ após o tratamento, segundo o autor pode estar associado ao balanço dos íons hidrogênio dos ácidos fracos presente na solução de $M$. oleífera com os íons hidróxido presentes na água residuária utilizada.

Nas Figuras 3 e 4são apresentados os resultados referentes à remoção de cor aparente durante o tempo de sedimentação para os ensaios com MO$\mathrm{KCl}$ e $\mathrm{MO}-\mathrm{NaCl}$, respectivamente. 


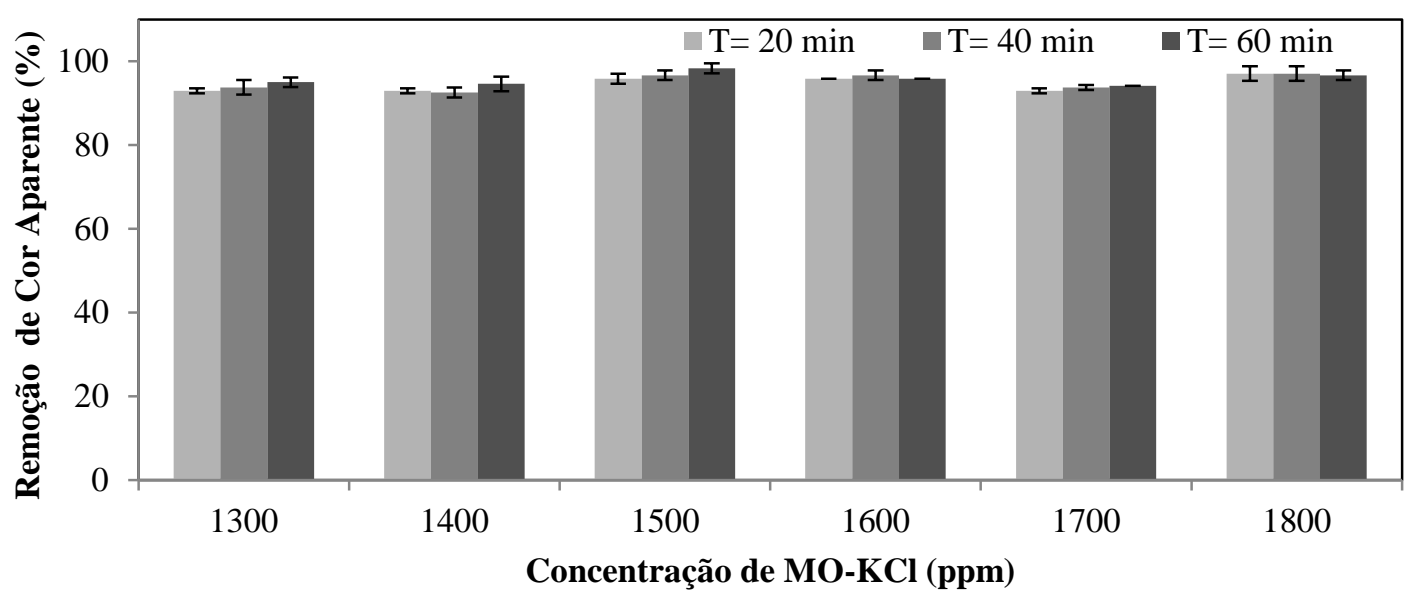

Figura 3. Remoção de Cor Aparente durante o tempo de sedimentação para MO-KCl.

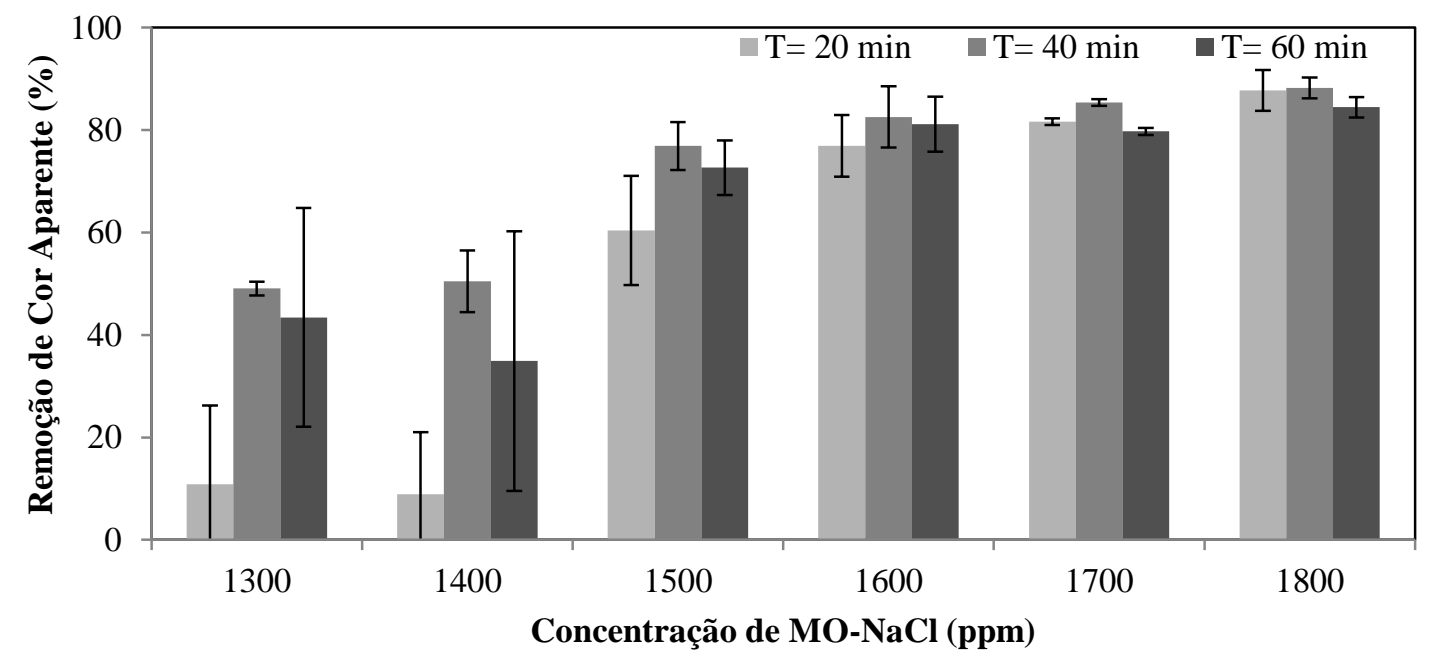

Figura 4. Remoção de Cor Aparente durante o tempo de sedimentação para MO-NaCl.

$\mathrm{Na}$ Figura 3 são apresentadas remoções de cor aparente superiores a 94\% um desvio-padrão muito pequeno.

$\mathrm{Na}$ Figura 4um incremento na remoção de cor aparente ocorreu após decorridos os tempos iniciais de sedimentação.

Resultados semelhantes aos de remoção de cor aparente foram encontrados para remoção de turbidez como pode ser observado nas Figuras 5 e 6.

Conforme consta nas Figuras 5 e 6, os melhores resultados de remoção de turbidez e os menores desvios-padrões são encontrados na Figura 5, ou seja, também para este parâmetro o coagulante extraído de $M$. oleíferacom solução de $\mathrm{KCl}$ 1,0 M alcançou melhores resultados.

Segundo Madrona et al. (2010), para o tratamento de água superficial de elevada turbidez o coagulante de $M$. oleífera extraído com $\mathrm{KCl}$ 1,0 M também apresentouos melhores resultados, ou seja, em pH 8,0 as melhores remoções de cor aparente e turbidez foram $82,0 \%$ e $96,0 \%$, respectivamente. 


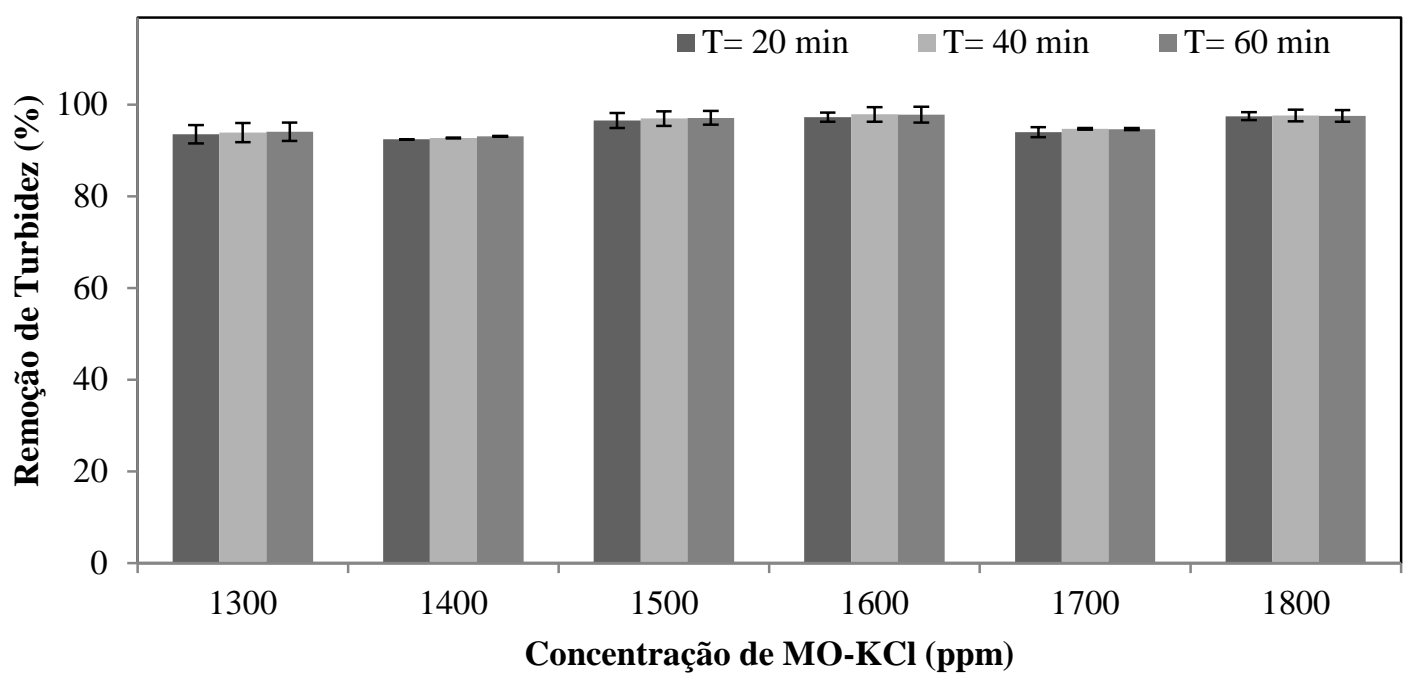

Figura 5. Remoção de turbidez durante o tempo de sedimentação para MO-KCl.

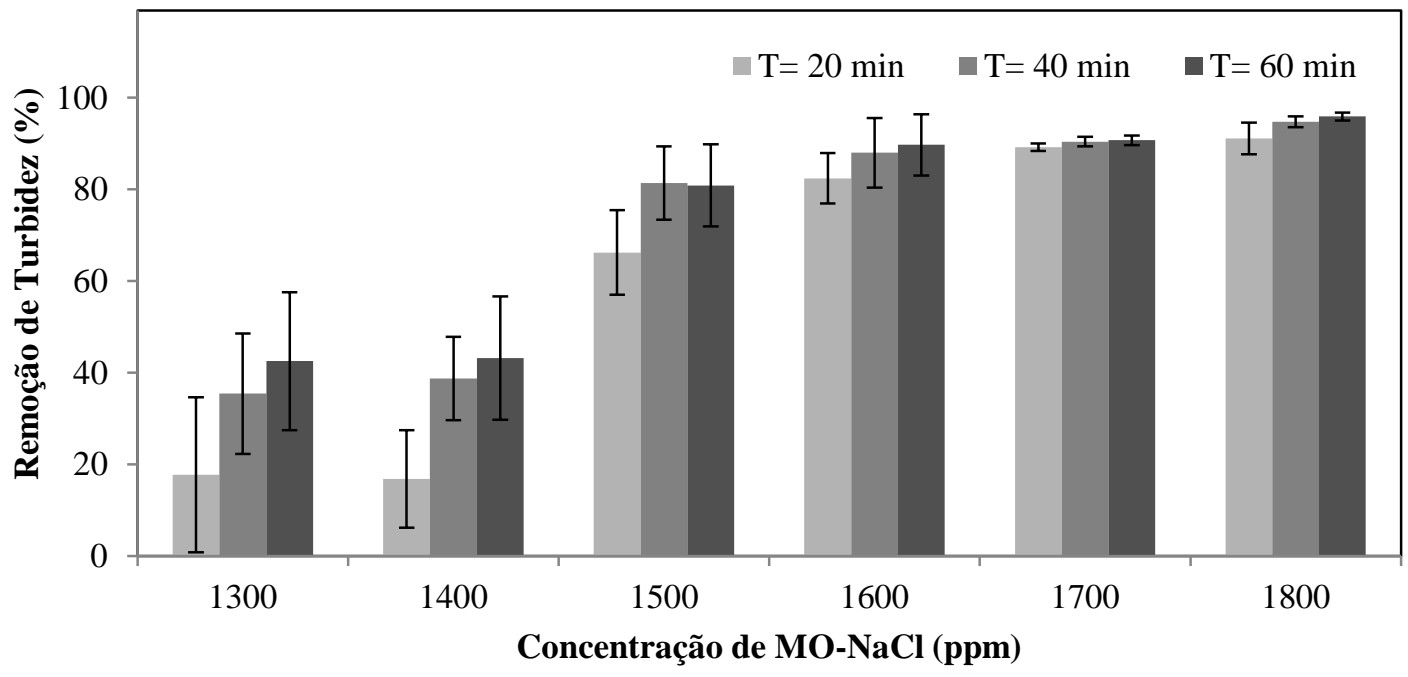

Figura 6. Remoção de turbidez durante o tempo de sedimentação para $\mathrm{MO}-\mathrm{NaCl}$.

As elevadas remoções de cor aparente e turbidez neste estudo eram esperadas, pois a água residuária da indústria de laticínios utilizada apresentou elevada concentração destes parâmetros. De acordo com Nkurunziza et al. (2009), o coagulante extraído da $M$. oleífera com solução salina se apresenta mais eficiente para águas superficiais com elevada turbidez, não sendo bom coagulante para águas com cor aparente e turbidez baixas.

$\mathrm{Na}$ Figura 6verificou-se que a remoção de turbidez foi mais significativa apenas quando se empregou concentrações maiores de coagulante $\mathrm{MO}-\mathrm{NaCl}$.

A observação das Figuras 3, 4, 5 e 6 permite afirmar que os desvios padrões identificados pelas barras de erro inseridas nestas figuras foram mais acentuados nos ensaios com MO$\mathrm{NaCl}$,principalmente nos tempos iniciais. Da mesma forma, é possível afirmar ainda que $\mathrm{MO}-\mathrm{KCl}$ apresentou resultados melhores e mais uniformes que MO$\mathrm{NaCl}$.

A avaliação das eficiências das soluções coagulantes extraídas com soluções salinas distintas e a determinação de qual seria a melhor 
solução coagulante para a água residuária da indústria de laticínios estão entre os objetivos do presente estudo.

As observações apresentadas por Madrona et al. (2010) em seus estudos para tratamento de água superficial de elevada turbidez são importantes, pois os autores observaram que o declínio na concentração salina teve relação com a diminuição nos percentuais de remoção de turbidez. O maior índice de proteína encontrado pelos autores foi na solução com $\mathrm{KCl}$ 1,0 M (23.400 mg L $\left.{ }^{-1}\right)$, sendo que este índice foi diminuindo conforme se utilizaram concentrações salinas mais baixas $\left(13.650 \mathrm{mg} \mathrm{L}^{-1}\right.$ para $0,1 \mathrm{M}$ e 9.750 $\mathrm{mg} \mathrm{L}^{-1}$ para $0,001 \mathrm{M}$ ). A extração do coagulante apenas com água destilada foi a que obteve o menor índice de proteína $\left(873 \mathrm{mg} \mathrm{L}^{-1}\right)$. Os autores verificaram que quanto maior o índice de proteína melhor é a eficiência na remoção de cor e turbidez.

$\mathrm{O} \mathrm{pH}$ da água residuária objeto deste estudo não foi ajustado $\mathrm{e}$ se manteve na faixa de 7,0 a 7,5 e isso também pode justificar as altas remoções para cor aparente e turbidez obtidas no ensaio com $\mathrm{MO}-\mathrm{KCl}$, já que Madrona et al. (2010) encontraram os melhores resultados com o pH 8,0, enquanto que no $\mathrm{pH} 4,0$ e 6,0 as remoções foram significativamente menores.

$\mathrm{Na}$ Figura 7são destacadas as remoções para o parâmetro DQO, observadas nos ensaios com $\mathrm{MO}-\mathrm{KCl}$ e $\mathrm{MO}-\mathrm{NaCl}$.

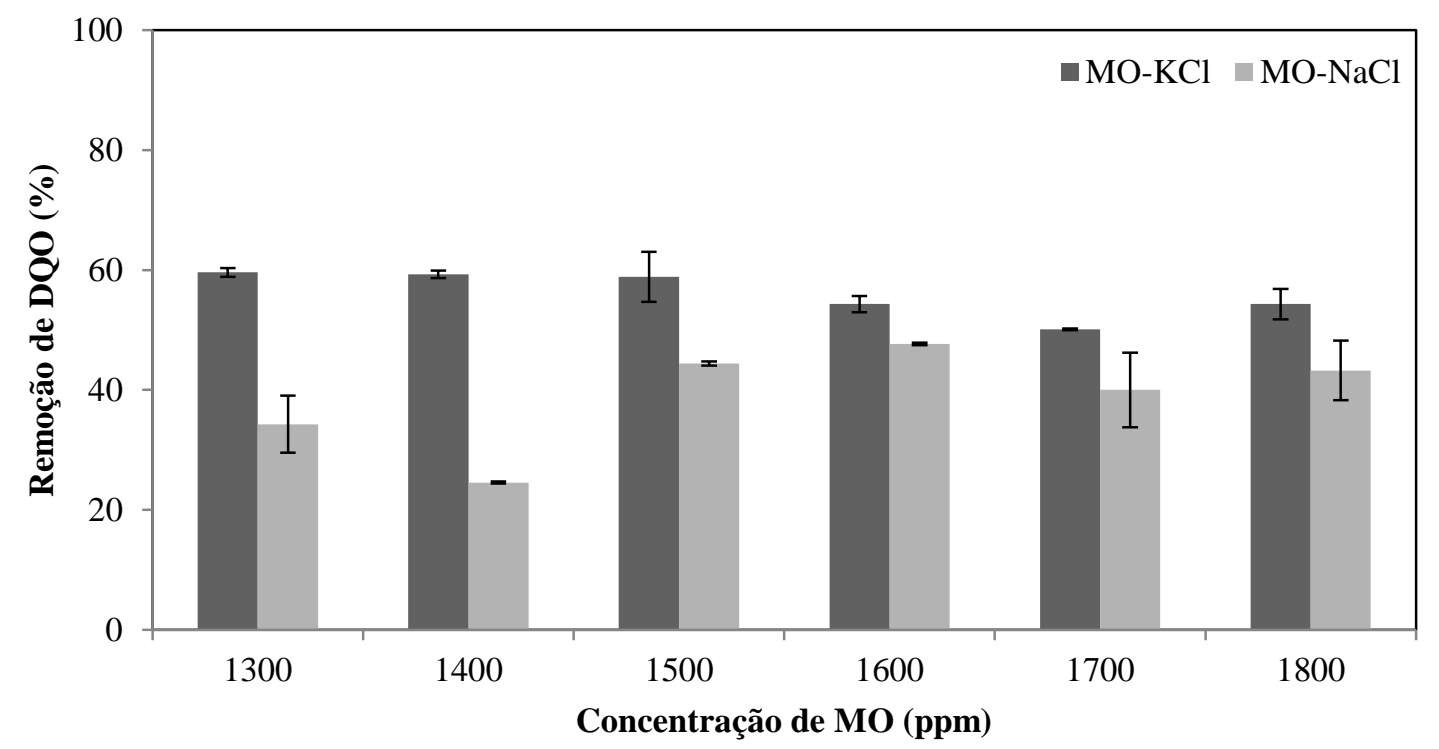

Figura 7. Remoção de DQO após 60 min de sedimentação utilizando MO-KCl e MO-NaCl em diferentes concentrações.

Ao observar a Figura 7 e comparar as remoções de DQO que utilizaram MO$\mathrm{KCl}$ e $\mathrm{MO}-\mathrm{NaCl}$ é possível constatar que a remoção de DQO ao utilizar $\mathrm{MO}-\mathrm{KCl}$ foi superior em todas as concentrações, nas quais, foi possível obter até $59,6 \%$ de remoção para o parâmetro DQO na concentração de 1300 ppm de MO-KCl. A melhor remoção de DQO com MO$\mathrm{NaCl}$ foi de $47,7 \%$ para a concentração de 1600 ppm.
Um estudo semelhante realizado por Sarkar et al. (2006) também obteve boa remoção de DQO. Neste estudo, os autores utilizaram o biopolímero quitosana para tratamento por coagulação/floculação de água residuária da indústria de laticínios. Os resultados deste estudo em termos de remoção de DQO para as concentrações entre 10 e 50 $\mathrm{mgL}^{-1}$ de quitosana e para o $\mathrm{pH} 4,0$ foram de $57 \%$ e são similares aos encontrados no ensaio com $\mathrm{MO}-\mathrm{KCl}$ para 
a concentração de 1500 ppm, no qual a remoção de DQO chegou a 58,9\%. Aparentemente a diferença entre as concentrações dos dois coagulantes aponta uma vantagem para o uso de quitosana, mas o custo deste coagulante é muito superior ao da $M$. oleífera e nos ensaios realizados com $M$. oleífera não foi necessário o ajuste de $\mathrm{pH}$, o que representa uma vantagem financeira $\mathrm{e}$ operacional importante.

Okuda et al. (1999) em seus estudos não observarem diferenças significativas quanto à eficiência na remoção de turbidez de amostras de água turva (sintética) com a utilização do coagulante $M$. oleífera extraída com diferentes sais $\left(\mathrm{NaCl}, \mathrm{KCl}, \mathrm{KNO}_{3}\right.$ e $\mathrm{NaNO}_{3}$ ); no presente estudo o melhor desempenho pode ser verificado nas Figuras 3, 4, 5, 6 e 7,para o coagulante de M. oleífera extraído com solução de $\mathrm{KCl}$ $1,0 \mathrm{M}$ em todas as concentrações utilizadas e em todos os parâmetros analisados.

A origem da água que foi tratada (água superficial ou residuária) pode influenciar nos resultados, ou seja, pela alta carga orgânica presente nas águas residuárias da indústria de laticínios, a dispersão do coagulante $\mathrm{MO}-\mathrm{KCl}$ causou maior desestabilização das partículas coloidais e posterior formação de grandes flocos do que o coagulante $\mathrm{MO}-\mathrm{NaCl}$, o que justificaria o comportamento diferente em relação aos estudos de Okuda et al. (1999).

Prasad (2009), ao tratar água residuária de destilariade álcool empregou diferentes sais $(\mathrm{NaCl}, \mathrm{KCl}$, $\mathrm{NH}_{4} \mathrm{Cl}, \mathrm{NaNO}_{3}$ e $\mathrm{KNO}_{3}$ ) para a extração do coagulante de $M$. oleíferae obteve os melhores resultados quando utilizou $\mathrm{NaCl}$ e KCl. As remoções de cor obtidas pelos autores alcançaram 56\% e 67\% para os sais $\mathrm{NaCl}$ e $\mathrm{KCl}$, respectivamente, ou seja, semelhante a este estudo obtiveram melhores resultados ao utilizar o sal $\mathrm{KCl}$ para extração do coagulante de M. oleifera.

\section{CONCLUSÃO}

Com base no estudo realizado conclui-se que:

- o uso do coagulante de $M$. oleífera não altera o $\mathrm{pH}$ das águas tratadas o que evita a necessidade de correção de pH após o tratamento;

- a extração do coagulante de $M$. oleífera com as soluções salinas concentradas $(1,0 \mathrm{M})$ possibilitou alcançar elevadas remoções de cor aparente $(98,3 \%)$ e turbidez (97,8\%), a remoção de DQO foi menor, mas não menos importante, pois chegou a 59,6\%;

- o coagulante de $M$. oleífera extraído com solução salina de $\mathrm{KCl}$ 1,0 $\mathrm{M}$ foi mais eficiente em termos de remoção de cor aparente, turbidez e DQO para a água residuária de laticínios do que o coagulante extraído com $\mathrm{NaCl}$ 1,0 M;

- a melhor concentração empregada no presente estudo foi $1500 \mathrm{ppm}$ de $\mathrm{MO}-\mathrm{KCl}$, pois apresentou a mais elevada remoção de cor aparente $\quad(98,3 \%$ seguido de 96,7\% em 1800 ppm), além das remoções de turbidez e DQO ( $97,1 \%$ e $58,9 \%$, respectivamente) estarem muito próximas das maiores remoções já destacadas;

- a M. oleífera se apresenta como uma importante alternativa aos coagulantes inorgânicos que têm desvantagens como a geração de grandes volumes de lodo inorgânico. AM. oleífera além de ser biodegradável também possui baixo custo.

\section{5. \\ BIBLIOGRÁFICAS}

REFERÊNCIAS

APHA. Standard Methods for the Examination of Water and Wastewater, New York: American Public Health Association, 1994.

BHATIA, S.; OTHMAN, Z.; AHMAD, A.B., 2007. Pretreatment of palm oil mill effluent (POME) using Moringaoleifera 
seed's as natural coagulant. Journal of Hazardous Materials. 145, pp. 120-126.

BHUPTAWAT, H.; FOLKARD, G.K.; CHAUDHARI, S., 2007.Innovative physico-chemical treatment of wastewater incorporating Moringaoleiferaseed coagulant. Journal of Hazardous Materials.142, pp. 477482.

DANALEWICH, J.R.; PAPAGIANNIS, T.G.; BELYEA, R.L.; TUMBLESON, M.E.; RASKIN, L., 1998. Characterization of dairy waste streams, current treatment practices, and potential for biological nutrient removal.Water Research, 12 (32), pp.3555-3568.

GALAMBOS, I.; MOLINA, J.M.; JÁRAY, P.; VATAI, G.; BEKÁSSYMOLNÁR, E., 2004. High organic content industrial wastewater treatment by membrane filtration. Desalination. 162, pp. 117-120.

HEREDIA, J.B.; SÁNCHEZ-MARTÍN, J., 2009.Removal of sodium lauryl sulphate by coagulation/flocculation with Moringaoleiferaseed extract. Journal of Hazardous Materials. 164, pp. 713-719.

KATAYON, S.; NOOR, M.J.M.M.; TAT, W.K.; HALIM, G.A.; THAMER, A.M.; BADRONISA, Y., 2007. Effect of natural coagulant application on microfiltration performance in treatment of secondary oxidation pond effluent. Desalination. 204, pp. 204-212.

KUSHWAHA, J.P.; SRIVASTAVA, V.C.; MALL, I.D., 2010.Treatment of dairy wastewater by commercial activated carbon and bagasse fly ash: Parametric, kinetic and equilibrium modelling, disposal studies. Bioresource Technology.10 (101), pp. 3474-3483.

MADRONA, G.S.; SERPELLONI, G.B.; VIEIRA, A.M.S.; NISHI, L.; CARDOSO, K.C.; BERGAMASCO, R., 2010. Study of the effect of saline solution on the extration of the Moringaoleifera seed's active component for water treatment. Water Air Soil Pollution.14 (211), pp. 409-415.

MUYIBI, S.A.; EVISON, L.M., 1995. Moringaoleifera seed's for softening hard water. Water Research. 4 (29), pp. 10991105.

NDABIGENGESERE, A; NARASIAH, K.S., 1998.Quality of water treated by coagulation using Moringaoleifera seeds. Water Research. 32, pp. 781-791.

NDABIGENGESERE, A; NARASIAH, K.S.; TALBOT, B. G., 1995.Active agents and mechanism of coagulation of the turbid water using Moringaoleifera.Water Research. 2 (29), pp. 703-710.

NKURUNZIZA, T.; NDUWAYEZU, J. B.; BANADDA, E. N.; NHAPI, I., 2009. The effect of turbidity levels and Moringaoleifera concentration on the effectiveness of coagulation in water treatment. Water Science \& Technology.59, pp. 1551-1558.

OKUDA, T.; BAES, A. U.; NISHIJIMA, W.; OKADA, M., 1999.Improvement of extraction method of coagulation active components from Moringaoleifera seed. Water Research. 33, pp. 3373-3378.

PRASAD, R. K., 2009.Color removal from distillery spent wash through coagulation using Moringaoleiferaseeds: Use of optimum response surface methodology. Journal of Hazardous Materials. 165, pp. 804-811.

RAMJEAWON, T., 2000. Cleaner production in Mauritian cane-sugar factories. Journal of Cleaner Production. 8 , pp. 503-510.

RENAULT, F.; SANCEY, B.; BADOT, P.M.; CRINI, G., 2009. Chitosan for coagulation/flocculation processes - An eco-friendly approach. European Polymer Journal.25, pp. 1337-1348. 
SARKAR, B.; CHAKRABARTI, P.P.;VIJAYKUMAR, A.; KALE, V., 2006. Wastewater treatment in dairy industries - possibility of reuse.Desalination. 195, pp. 141-152.

SCHMITT, D. M. F.; FAGUNDESKLEN, M. R.; VEIT, M. T.; BERGAMASCO, R., 2012. Tratamento de águas residuárias da indústria de laticínios pelo processo de coagulação/floculação utilizando a semente de Moringa oleifera. Potencialidades da Moringa oleifera Lam. Cap. 24, v. 2, Editora UFS, pp. 301-316.

SENGIL, I. A.; ÖZACAR, M., 2006. Treatment of dairy wastewaters by electrocoagulation using mild steel electrodes. Journal of Hazardous Materials, B 137, pp. 1197-1205.

SILVA, C. A.; SILVEIRA, C.; SILVA, F. A.; FAGUNDES-KLEN, M. R.; BERGAMASCO, R., 2012. Classificação dos lodos formados durante o processo de coagulação/floculação da água com os coagulantes PAC eMoringa oleifera. Engevista. 14 (3), pp. 302-309.

TCHAMANGO, S.; NANSEU-NJIKI, C.P.; NGAMENI, E.; HADJIEV, D.; DARCHEN,A., 2010. Treatment of dairy effluents by electrocoagulation using aluminium electrodes. Science of the Total Environment.408, pp. 947-952. 